Itinéraires Itinéraires

Littérature, textes, cultures

2017-2 | 2018

Féminismes quatrième génération

\title{
L'électoralisation du féminisme : un nouvel antiféminisme ordinaire
}

Electoralization of Feminism: A New Ordinary Anti-Feminism

Stéphanie Pahud

\section{OpenEdition}

Journals

Édition électronique

URL : http://journals.openedition.org/itineraires/3804

DOI : $10.4000 /$ itineraires.3804

ISSN : 2427-920X

Éditeur

Pléiade

Référence électronique

Stéphanie Pahud, «L'électoralisation du féminisme : un nouvel antiféminisme ordinaire », Itinéraires [En ligne], 2017-2 | 2018, mis en ligne le 10 mars 2018, consulté le 02 mai 2019. URL : http:// journals.openedition.org/itineraires/3804; DOI : 10.4000/itineraires.3804

Ce document a été généré automatiquement le 2 mai 2019.

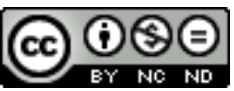

Itinéraires est mis à disposition selon les termes de la licence Creative Commons Attribution - Pas d'Utilisation Commerciale - Pas de Modification 4.0 International. 


\title{
L'électoralisation du féminisme : un nouvel antiféminisme ordinaire
}

\author{
Electoralization of Feminism: A New Ordinary Anti-Feminism
}

\section{Stéphanie Pahud}

\begin{abstract}
Une fois précisé (et cela est difficile) un certain nombre de libertés très concrètes à conquérir qui sont autant d'obstacles à abattre pour commencer à vivre, reste la liberté, la folle liberté de donner forme à son destin, l'affolante liberté de donner sens à l'aberration de la vie. Et je défierai qui que ce soit de prouver en quoi, sur ce point, les femmes seraient moins concernées que les hommes, même si le sens commun laisse à penser que donner la vie dispense amplement de chercher à donner sens à

sa vie.

Annie Le Brun, « Une obscure utopie », 1990, p. 254.
\end{abstract}

\section{Liminaires}

1 Je suis féministe et je n'éprouve pas le besoin d'accompagner cet attribut de précautions oratoires. Il m'a cependant fallu beaucoup de lectures, de temps, de dialogues et d'expériences surtout, pour être au clair avec mon rapport à ce mot et à ses différents investissements, pour qu'il ne stagne pas au stade d'emprunt vacillant. Il me faudra encore beaucoup de lectures, de temps, de dialogues et d'expériences surtout pour trouver des mises en discours (et en corps) de mon rapport à ce mot et de ma manière de l'investir qui n'induisent pas de sensations d'engagement vacillant.

2 Le féminisme n'est pas un costume prêt-à-passer, mais un état à incorporer, à négocier. Mouvement à la fois politique, culturel, social, historique, militant et intellectuel, il s'incarne en ce début de $\mathrm{xxI}^{\mathrm{e}}$ siècle dans une diversité croissante de tendances, dont la 
partialité des interprétations, biaisées par leur contexte d'émergence, est notamment visibilisée par un rendu médiatique extrêmement confusionnel :

«Femen partout, féminisme nulle part », titrait ainsi Mona Chollet dans un article $\mathrm{du}$ «Monde diplomatique», en 2013. [...] Une impression d'éparpillement des collectifs qui, en outre, participe au flou artistique. Les Femen, La Barbe, Osez le féminisme, Georgette Sand, Les chiennes de garde... « Il y a une multiplication de mouvances à l'heure actuelle, mais plus de mouvement général de société ", constate Patricia Roux. C'est ce que déplorait la féministe Marie-Josèphe Bonnet en 2014, alors interrogée par un journaliste du magazine français « Marianne »: «La confusion est très grande aujourd'hui sur les objectifs, les désirs, les politiques féministes. On s'aperçoit qu'on ne pense pas toutes pareil ? C'est évident. Il y a une désertification? C'est inévitable. » (Femina, 6 mars 2016)

3 Symétriquement, l'antiféminisme se déploie dans une diversité croissante de tendances, dont la compréhension aléatoire est de même visibilisée par un rendu médiatique tout aussi confusionnel et idéologiquement hybride. Pour exemple, fin 2014, le mot « féminisme » a été sélectionné dans la liste des « mots à bannir en 2015 » par le magazine d'information américain Time:

feminist: You have nothing against feminism itself, but when did it become a thing that every celebrity had to state their position on whether this word applies to them, like some politician declaring a party? Let's stick to the issues and quit throwing this label around like ticker tape at a Susan B. Anthony parade ${ }^{1}$.

Le Time s'est cependant ravisé suite à la polémique soulevée par son choix :

TIME apologizes for the execution of this poll; the word 'feminist' should not have been included in a list of words to ban. While we meant to invite debate about some ways the word was used this year, that nuance was lost, and we regret that its inclusion has become a distraction from the important debate over equality and justice ${ }^{2}$.

Six mois plus tard, en France, le numéro 26 du mensuel polémiste Causeur affichait en Une «La terreur féministe. Sexisme, inégalités, harcèlement... Elles vous ont à l'œil », avec pour image une femme lookée fifties tenant une tronçonneuse dans les mains : 
Fig. 1. Une du numéro 26 de Causeur

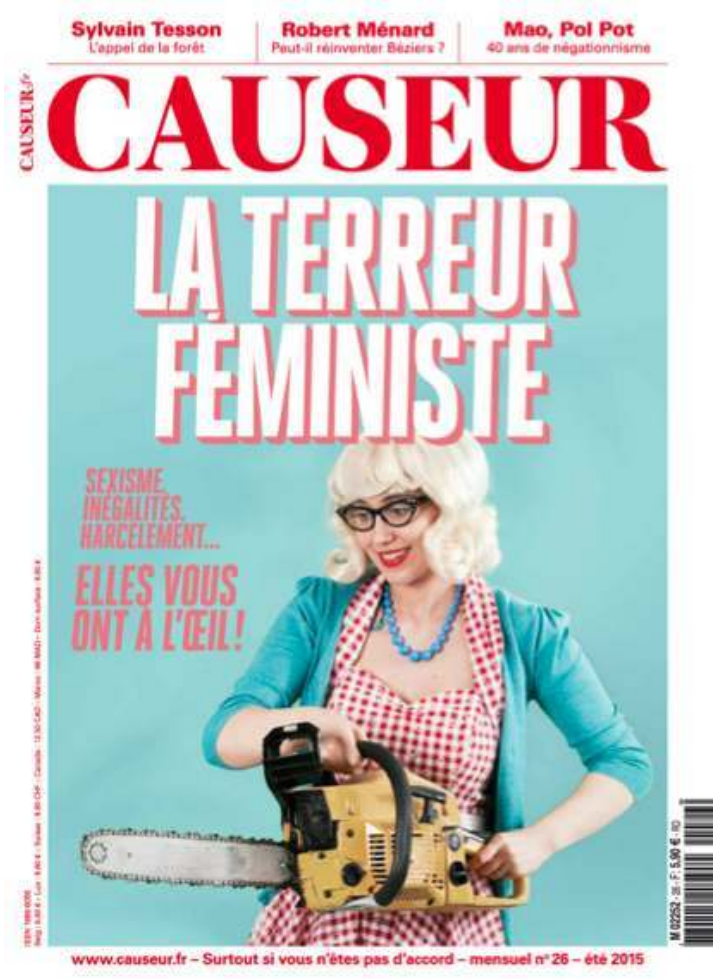

5 En Suisse, au printemps 2016, en Une de l'édition citée plus haut de l'hebdomadaire Femina, on pouvait lire « Nouveau tabou. Qui veut la peau du féminisme? » et l'on était invité à mesurer dans l'éditorial le degré de déconsidération du terme, ayant pris l'allure, " même chez les féministes elles-mêmes », d'une " patate chaude » :

Comment parler du féminisme sans déclencher de réflexe gerbatoire, de litanie approximative, de moues convenues? Puisque, dans un monde qui s'apprête à basculer dans le 3.0, le féminisme est au mieux une distraction pour dilettantes, au pire une insulte. (Femina, éditorial d'Adelita Genoud, 6 mars 2016, p. 3)

En février 2017, le $444^{\mathrm{e}}$ numéro du mensuel féminin français Biba affichait quant à lui « Wonder woman en a ras la cape »:

Stoooop, on en a marre de jouer les super women! Trop fatigant, trop culpabilisant... C'est décidé, on lâche prise et on kiffe !!! Vous avez remarqué à quel point nous, les femmes, on s'en demande beaucoup trop? On veut toujours tout bien faire, on gère tout toute seule, on assume tout. Mais meeeerde, qui a dit que ça ne pouvait marcher que comme ça? Et si on lâchait du lest, si on arrêtait de tout faire, de courir après la double journée ? Mode d'emploi pour y arriver (ou au moins essayer !). (Anne Ulpat)

7 Et il semble désormais d'usage dans les interviews contemporaines tant de questionner la « ringardise » du féminisme que de justifier sa mobilisation. En guise d'illustration, cette question posée par un hebdomadaire suisse à une ancienne conseillère fédérale active dans la lutte pour l'égalité des sexes depuis plusieurs décennies :

Le féminisme n'apparait-il tout de même pas un peu ringard aujourd'hui?

Non au contraire. Le terme de féminisme a toujours eu de la peine à s'imposer très largement. Il y a toujours eu des femmes qui craignaient de s'afficher comme féministes. Cela a accompagné l'histoire de la longue marche des femmes vers l'émancipation et l'égalité des droits. [...] Moi, je me dis volontiers féministe. Je me 
suis dite aussi suffragette à l'époque où on n'avait pas les droits politiques. Car j'aime m'inscrire dans la continuité de cette lutte. Mais ce qui m'importe plus que les mots, c'est la justesse de nos revendications et la force qui est en nous pour lutter en vue de les obtenir. (Ruth Dreifuss, Le Matin Dimanche, 26 mars 2017)

Comme le synthétise Guionnet, «il existe actuellement un véritable malaise de l'opinion publique par rapport au féminisme» (2017: 117). Cette contribution, située (Haraway 1988, Anderson 2015) puisqu'elle présuppose que ma performance singulière du féminisme participe de la dé-limitation et de la couleur des métadiscours que je produis, est une occasion d'argumenter que pour comprendre ce malaise, il serait fécond de sortir de la volonté de « définir le féminisme » pour aller vers une description des « rapports au féminisme ", la notion de « rapport à », définie par Barré-De Miniac, "suggér[ant] l'idée d'une orientation ou disposition de la personne à l'égard d'un objet [...] et à l'égard de la mise en œuvre pratique de cet objet dans la vie personnelle, culturelle, sociale et professionnelle» ([2000] $2015: 15)$. On peut appliquer par analogie au féminisme ce que Barré-de Miniac dit de l'écriture :

S'agissant [du féminisme], outil symbolique, inscrit si profondément dans l'histoire individuelle de chaque [individu] autant que dans l'histoire collective de la société et des groupes auxquels il appartient, son usage, balbutiant lors de [l'incorporation] autant qu'expert par la suite, ne peut être compris que situé dans le réseau complexe des dimensions marquantes de ces histoires individuelle et collective. À l'histoire il convient d'adjoindre le présent des situations [de féminisme], tel qu'il peut être vécu, perçu, plus ou moins bien compris, analysé plus ou moins consciemment par [l'individu]. Globalement donc, le rapport [au féminisme] désigne des conceptions, des opinions, des attitudes, de plus ou moins grande distance, de plus ou moins grande implication, mais aussi des valeurs et des sentiments attachés [au féminisme], à son [incorporation] et à ses usages. (D'après Barré-De Miniac [2000] 2015 : 15-16)

Nous émettons l'hypothèse que les mises en discours «féministes » ou " antiféministes » ne nous renseignent pas tant sur le féminisme ou l'antiféminisme que sur les rapports que certains individus entretiennent avec les idées qui s'y rattachent.

10 Nous émettons corrélativement l'hypothèse qu'en termes de rapport au féminisme, l'on assiste en ce début de $\mathrm{xxI}^{\mathrm{e}}$ siècle à une "électoralisation" de ce dernier: nous empruntons le concept à Gracq, qui l'a employé en 1950 relativement à la littérature pour désigner « un geste fiévreux d'adhésion ou de non-adhésion, une sympathie ou une antipathie pures, et qui se traduit par exemple, dans les articles si fréquents maintenant, du type dit "coup de poing" » (1964: 102-103). Par le biais d'une analyse discursive de quelques positionnements féministes et antiféministes, cette contribution, qui fera dialoguer théorie du discours, linguistique textuelle et études de genre, souhaite ainsi questionner quelques rapports au féminisme et contribuer à une désévidentialisation de son appropriation ordinaire.

\section{L'antiféminisme, consubstantiel au féminisme}

11 Comme le suggère Rochefort, il est intéressant d'ouvrir le débat « sur le sens historique et la réception du féminisme » $(1998: 147)$. L'histoire du terme dans son sens moderne est, à l'origine, celle d'une réappropriation à partir d'un adjectif stigmatisant les hommes. L'étiquette féministe a en effet été créée en 1870 par le corps médical pour désigner un arrêt de développement et un défaut de virilité chez les sujets masculins ${ }^{3}$ : 
Nous avons tous connu, pendant les années de notre enfance et plus tard grandissant avec nous, des enfants, des adolescents, puis des hommes, qui ne subissaient pas les mêmes transformations que les diverses étapes de l'âge amenaient chez leurs camarades : c'est ainsi que, enfants, ils ressemblaient pour ainsi dire plus à des filles qu'à des garçons; adolescents, ils ressemblaient à des enfants; hommes, ils n'étaient qu'adolescents; chez quelques-uns même, le caractère et l'esprit, les tendances passionnelles, les aptitudes sociales, non moins que les apparences corporelles, présentaient une sorte de caractère féminin que nous appellerons le féminisme, si vous le voulez. Ce n'est point là la monstruosité vraie, ce n'est point l'arrêt de développement total d'un organe; en un mot, ce n'est pas l'hermaphrodisme, c'est une transition, un intermédiaire qui n'est pas assez saisissable au premier abord pour fixer l'attention des savants, et c'est ainsi qu'on explique que cet intéressant cas d'histoire naturelle ait échappé jusqu'à présent aux naturalistes. (P. Lorain, décembre 1870, dans Du féminisme et de l'infantilisme chez les tuberculeux, Ferdinand-Valère Fanneau de la Cour, thèse pour le doctorat en médecine présentée et soutenue le 18 janvier 1871, Paris, A. Parent)

En 1872, le terme féministe fait sa première apparition publique sous la plume de Dumas fils dans L'Homme-femme, un essai visant à démontrer la nécessité de châtier une femme adultère par la peine capitale. Le romancier s'approprie le terme pour marquer son opposition à ce que ce néologisme qualifiant une catégorie d'hommes favorables à la cause des femmes présuppose en termes de conception des rôles de sexe et de genre :

Les féministes, passez-moi ce néologisme, disent, à très-bonne intention d'ailleurs : Tout le mal vient de ce qu'on ne veut pas reconnaître que la femme est l'égale de l'homme et qu'il faut lui donner la même éducation et les mêmes droits qu'à l'homme; l'homme abuse de sa force, etc., etc. Vous savez le reste. Nous nous permettrons de répondre aux féministes que ce qu'ils disent là n'a aucun sens. La femme n'est pas une valeur égale, supérieure ou inférieure à l'homme, elle est une valeur d'un autre genre, comme elle est un être d'une autre forme et d'une autre fonction. La preuve qu'elle n'est pas aussi forte que l'homme, c'est qu'elle se plaint toujours de ce que l'homme est plus fort qu'elle; or, si la nature a donné la force à l'homme, c'est pour qu'il s'en serve, comme il doit se servir de tous les dons qu'il a reçus pour l'œuvre qu'il a à faire. En effet, un des premiers usages que le masculin a fait de sa force a été d'enfermer et de subordonner le plus possible le féminin, dont il a besoin dans certains cas, s'étant aperçu qu'il lui en coûte cher, à lui masculin, quand ce féminin est en liberté, même dans un paradis (91-93).

13 L'idée de fond qui sous-tend cette première application de l'étiquette à une manière littéralement insensée de penser, est que le féminisme "dénature » les sexes et que la libération des femmes compromet les rapports harmonieux que les unes et les autres devraient entretenir. Dumas fils explicite ces dérives du féminisme quelques paragraphes plus loin dans L'Homme-femme :

Ce n'est [...] pas parce que nous lui donnons l'éducation qu'elle reçoit que la femme est ce qu'elle est, c'est parce qu'elle est ce qu'elle est que nous lui donnons l'éducation qu'elle reçoit; et quand elle se prétend capable d'édicter des lois, de commander des armées et de conduire des locomotives, elle est aussi ridicule que le serait le sexe fort s'il voulait porter des chignons, montrer ses épaules et allaiter des enfants. Vouloir réunir les deux natures en une seule, ce serait l'hermaphrodisme, qui est l'impuissance mâle et femelle. (94)

14 Comme le résume Preciado dans une chronique intitulée " Féminisme amnésique », « les premières féministes ont donc été des hommes: des hommes que le discours médical a considérés comme anormaux pour avoir perdu leurs "attributs virils" ( 2014 : en ligne). Il faut attendre que la suffragiste Hubertine Auclert s'approprie le terme pour que sa 
connotation s'inverse et qu'il soit employé pour exprimer un rapport empathique à la lutte des femmes :

On nous demande ce que nous pensons de la classification des hommes, dans une sphère, et de la classification des femmes dans une autre sphère. Eh bien! nous pensons à l'encontre de tous les masculinistes qui érigent les porte-barbe en dieux, que le sexe ne confère pas d'attributions particulières et que, si les Chinois sont sots d'imposer à l'homme et à la femme des rôles différents dans la société, les Européens qui se targuent d'être autrement civilisés que les Chinois, sont encore bien plus sots d'assigner à l'être humain, selon que le hasard de la naissance le fait homme ou femme, des attributions déterminées. (Hubertine Auclert, La Citoyenne. Journal des droits de la femme, $\mathrm{n}^{\circ} 54$, février 1882).

Mais cette resémantisation ne suffit pas pour laver le terme de ses usages antérieurs, de son « impopularité », et pour freiner la naturalisation du préjugé selon lequel féminisme et « féminité » seraient incompatibles. De nombreuses femmes mettront ainsi en discours un rapport de rejet, au mépris de leurs convictions, pour se défendre de certaines déviances prêtées au rôle. Comme le déplore la philosophe Fraisse, "le féminisme apparait comme un désordre, une passion, une hystérie, rarement comme un engagement raisonné dans l'espace politique »; il passe pour «rel[ever] de l'humeur et non de la réflexion » $(2008:$ : 54).

Dans les années 1970, le terme cristallise des tensions entre féministes de différents courants et à l'opposition entre féminisme et antiféminisme s'ajoute une opposition entre féminisme " émancipatoire » et féminisme « à contre-emploi ». Nous prendrons l'exemple de Le Brun. En 1977, l'auteure publie Lâchez tout, présenté comme "un appel à la désertion » :

[...] un appel à la désertion contre l'avachissement de la révolte féministe avec Simone de Beauvoir, contre le jésuitisme de Marguerite Duras et de Xavière Gauthier, contre le poujadisme de Benoîte Groult, contre le débraillé d'Annie Leclerc, contre les minauderies obscènes d'Hélène Cixous, contre le matraquage idéologique du chœur des vierges en treillis et des bureaucrates du M.L.F., désertez, lâchez tout. Le féminisme, c'est fini. (Texte de rabat de quatrième de couverture)

Le Brun déplore qu'aux XVIII et $\mathrm{XIX}^{\mathrm{e}}$ siècles on ait tenté d'effacer les différences et que dans les années 1970, on les réaffirme pour se réclamer d'un pouvoir dont les femmes auraient été privées :

J'ai peur que la vie de toutes celles qu'on a contraintes à être ce qu'elles ne sont pas, leur soit une nouvelle fois dérobée au nom de la Femme. N'y a-t-on pas réussi ailleurs ou avec d'autres indifféremment au nom de Dieu, de l'Homme, de l'Histoire, de la Révolution? Faut-il vraiment que les femmes accèdent à la liberté de devenir les victimes de cette nouvelle escroquerie? Faut-il vraiment que les femmes se laissent enfermer dans le ghetto d'une identification sexuelle définitive pour conquérir leur indépendance? Faut-il vraiment que chaque femme appauvrisse sa sensibilité en entérinant intimement cette spécialisation génitale qui n'a fait qu'ajouter à la misère des rapports humains? Telles sont les nouvelles questions politiques qui se posent aujourd'hui aux femmes. Jusqu'à quand consentiront-elles à ce que la bureaucratie néo-féministe mette tout en œuvre pour les empêcher d'y répondre? (Le Brun 1977 : 216-217)

18 Treize ans plus tard, dans " Retournement de jupe », Le Brun dénonce le renouveau néoféministe, un retour aux "valeurs sûres», le triomphe d'un conformisme répugnant rejetant tout ce qui n'est pas rationalisable, la dépassionnalisation et la volonté monstrueuse de fabriquer un homme nouveau : 
Je dis les femmes quand je devrais dire les néoféministes. Les femmes sont ailleurs. À voir leurs mouvements d'hermine dans le printemps gris-léger de Paris, j'imagine encore que rien n'est perdu. À elles, alors, de refuser que des femmes aujourd'hui - à la place des hommes hier - parlent en leur nom et que, sous des propos contraires à ceux d'il y a vingt ans, celles-ci continuent de les tromper sur leur liberté. (Le Brun 1990 : 29-30)

Présentée dans une exposition sur les «contre-cultures des années 1969 à 1989 » («L'esprit français ", Paris, Maison Rouge) comme "féministe à sa manière, et aussi politiquement engagée que celles qu'elle cible », Le Brun a eu l'occasion de résumer son rapport au féminisme lors d'une rencontre lausannoise autour de son œuvre, quarante ans après la publication de Lâchez tout :

Je n'avais pas besoin de Sade pour ma révolte contre le féminisme. J'étais révoltée, parce qu'au moment où les filles pouvaient commencer à réellement, à sérieusement penser leur liberté - c'était les débuts de la contraception, une révolution considérable - l'idée qu'elles ratent cette occasion-là, d'une certaine façon qu'elles trahissent le véritable combat qui avait été celui de certaines féministes de la fin du $18^{\mathrm{e}}$ siècle, certains personnages comme Olympe de Gouges, etc., et puis après les féministes du $19^{\mathrm{e}}$, les féministes anarchistes du début du $20^{\mathrm{e}}$ siècle, qu'on retourne, qu'on se rassemble pour se ressembler, que tout d'un coup on recommence, qu'il y ait une sorte de ghetto, qu'on bâtisse une idéologie pseudostalinienne, alors là, moi ça m'a mise dans tous les états, et dans ces cas-là, je n'ai besoin de personne. Là, je cogne. [...] Quand j'étais passée à la télévision, j'avais été invitée à Apostrophes, je ne voulais pas y aller, mais comme les féministes d'alors, bureaucratiques, avaient répandu le bruit que ça ne pouvait pas être une fille, que c'était un homme qui avait écrit ce texte-là, j'y suis allée pour montrer que... voilà, et c'est là que Jean-Jacques Pauvert m'a vue. C'était le moment où il y avait Gisèle Halimi, qui avait fait un programme commun des femmes (en plus ça faisait PCF), un des trucs phare c'était la censure : la censure dans les écoles, la censure dans la littérature. Alors là j'ai posé une question à savoir : qu'est-ce qu'un mouvement de libération qui commence par censurer? Nietzsche, Sade, Miller, etc. J'ai dit que c'était sans doute pour qu'on nous fasse lire Benoîte Groult, etc. Pauvert a été tellement content qu'une fille commence à dire sur la censure des choses comme ça, qu'il a tout fait pour que l'on se rencontre. Pour la violence, là, je n'avais pas besoin de Sade. (Annie Le Brun, rencontre du 23 mai 2017 animée par Quentin Mouron, Librairie de la Louve, Lausanne)

Le militantisme "au nom de la Femme» est également condamné, sous d'autres modalités, par Wittig pour qui «l'ambiguïté du terme féministe résume toute la situation » :

Que veut dire « féministe »? Féministe est formé avec le mot «femme » et veut dire "quelqu'un qui lutte pour les femmes ». Pour beaucoup d'entre nous, cela veut dire "quelqu'un qui lutte pour les femmes en tant que classe et pour la disparition de cette classe ». Pour de nombreuses autres, cela veut dire « quelqu'un qui lutte pour la femme et pour sa défense »- pour le mythe, donc, et son renforcement. Pourquoi a-t-on choisi le mot "féminisme ", s'il recèle la moindre ambiguïté ? Nous avons choisi de nous appeler "féministes ", il y a dix ans, non pas pour défendre le mythe de la femme ou le renforcer ni pour nous identifier avec la définition que l'oppresseur fait de nous, mais pour affirmer que notre mouvement a une histoire et pour souligner le lien politique avec le premier mouvement féministe. (Wittig [1980] $2013:$ :51-52)

21 Loin d'être conçues comme "antiféministes", ces mises en discours de rapports conflictuels à certaines déclinaisons du féminisme contribuent néanmoins à rendre poreuse la frontière entre les postures, souvent réduites à quelques traits caricaturaux. 
Les années 1990 voient naître quant à elles un rejet des fondements même du féminisme, dont la pertinence des conquêtes échapperait aux nouvelles générations (Aronson 2015). Badinter explique dans son essai, Le Conflit. La femme et la mère ce tournant idéologique :

Filles de mères féministes, militantes ou non, elles ont procédé au règlement de compte classique des filles à l'égard des mères. Après les remerciements d'usage pour la contraception et l'avortement, c'est un constat d'échec qui est établi. Échec des mères que les filles ne veulent pas imiter et que l'on peut résumer ainsi : vous avez tout sacrifié pour votre indépendance et au lieu de cela, vous assumez la double journée de travail, vous êtes sous-estimées professionnellement et au bout du compte vous êtes perdantes sur tous les fronts. Au-delà de cette critique, c'est l'étiquette « féministe » qui fut rejetée, comme si elle donnait une image détestable des femmes. La nouvelle génération reprit à son compte les stéréotypes machistes les plus éculés, qui associent les féministes à l'hystérie, l'agressivité, la virilité et la haine des hommes. Le jugement fut sans appel : ringard. (Badinter 2010 : 161-162)

De nouvelles étiquettes sont alors inventées pour symboliser non pas d'«autres " féminismes, mais d'autres rapports au féminisme. Les femmes décrites par Badinter, qui affirment qu'elles choisissent librement d'adopter les comportements stéréotypiquement attribués au féminin, se revendiquent volontiers d'un "post-féminisme »-le concept étant régulièrement investi depuis les années 1980 pour signifier tantôt une « mort » du féminisme, tantôt une égalité prétendument réalisée, tantôt la naissance d'un nouveau cycle féministe (pour une synthèse, voire Aronson 2015) -, ou d'un féminisme "profemme ", lequel valorise maternité, hétérosexualité et féminité stéréotypée (Dupuis-Déri et Lamoureux 2015). Comme le souligne Macé (2003), ces étiquettes masquent en l'occurrence un antiféminisme culturel qui, loin de libérer les femmes, les rend simplement responsables de leur trajectoire sociale. Le développement d'une «culture postféministe » contribue à brouiller les frontières. Fakhry (2012) montre par exemple que les films d'horreur féminins considèrent les femmes au foyer comme des victimes du système patriarcal, mais ne font pas pour autant l'éloge des femmes qui travaillent. Au contraire, ils montrent que leur émancipation les conduit à leur perte et à des défaillances maternelles notamment (2012: 75). Cossy et al. décrivent cette relation paradoxale de certains objets culturels aux féminismes et aux patriarcats :

Quelles que soient la vertu politique et la valeur utopique qu'on lui accorde, le postféminisme ainsi entendu remet alors en question l'une des oppositions fondatrices du féminisme universaliste moderne, celle entre le féminisme et le féminin hétéronormé naturalisé - dénoncé comme une construction sociale à déconstruire. [...] Le féminin, quand il est cité, pastiché ou parodié ou encore resignifié, ne fonctionne pas comme une ligne de partage immédiat ou indiscutable entre le féminisme et son envers, l'hétéronormativité naturalisée. Il ré-ouvre au contraire un espace au sein duquel les rapports entre "féminisme » et "féminin " sont redéfinis. (Cossy et al. 2009 : 10-11)

Badinter formule le rôle que joue dans l'adoption de cette attitude post-féministe / antiféministe la peur de perdre son "identité spécifique», une peur d'être «désidentifié », de ne plus être « reconnu » socialement :

Jusqu'à hier les univers masculins et féminins étaient strictement différenciés. La complémentarité des rôles et des fonctions nourrissait le sentiment d'identité spécifique à chaque sexe. Dès lors qu'hommes et femmes peuvent assumer les mêmes fonctions et jouer les mêmes rôles - dans les sphères publiques et privées -, que reste-t-il de leurs différences essentielles? Si la maternité est l'apanage de la femme, est-il concevable de s'en tenir à une définition négative de l'homme : celui qui ne porte pas d'enfant? De quoi provoquer un profond vertige existentiel chez celui-ci... (2010 : 12-13) 
L'essai du journaliste politique Éric Zemmour, Le Premier Sexe, est emblématique d'une radicalisation masculine de ce rapport au féminisme induit par la peur de l'indifférenciation. Selon le journaliste, «à leur peur archaïque du phallus, du "viol de la pénétration", les femmes d'aujourd'hui répond[rai]ent par un malsain désir du même, une immense tentation lesbienne » (2006: 24). Le féminisme ne serait dès lors qu'« une immense machine à fabriquer du même » qui laisserait « une société du désordre [...] supplant[er] une société de l'ordre » (2006: 25 et 26$)$ :

C'est tout le paradoxe féminin. Les femmes conduisent quand la vitesse est limitée ; elles fument quand le tabac tue; elles obtiennent la parité quand la politique ne sert plus à grand-chose ; elles votent à gauche quand la Révolution est finie; elles deviennent un argument de marketing littéraire quand la littérature se meurt; elles découvrent le football quand la magie de mon enfance est devenue un tiroircaisse. Il y a une malédiction féminine qui est l'envers d'une bénédiction. Elles ne détruisent pas, elles protègent. Elles ne créent pas, elles entretiennent. Elles n'inventent pas, elles conservent. Elles ne forcent pas, elles préservent. Elles ne transgressent pas, elles civilisent. Elles ne règnent pas, elles régentent. En se féminisant, les hommes se stérilisent, ils s'interdisent toute audace, toute innovation, toute transgression. Ils se contentent de conserver. On explique en général la stagnation intellectuelle et économique de l'Europe par le vieillissement de sa population. Mais Cervantes écrivit Don Quichotte à soixante-quinze ans; de Gaulle revint au pouvoir à soixante-huit, et le chancelier allemand Adenauer à plus de soixante-dix. On ne songe jamais - ou on n'ose jamais songer - à sa féminisation. (Zemmour 2006 : 128-129)

\section{Une électoralisation du féminisme}

La circulation par voix médiatisées / médiatiques de rapports critiques au féminisme non situés, catalyse un antiféminisme discursivement construit comme aussi naturel que la binarité des sexes, un " antiféminisme ordinaire », banalisé :

Dans la conjoncture actuelle, par l'expression "antiféminisme ordinaire», je désigne les discours et les pratiques qui, sans nécessairement recourir à des interprétations fallacieuses extrémistes ou moralisantes, s'opposent, implicitement ou explicitement, aux projets portés par le féminisme et font obstacle aux avancées des femmes dans les différents domaines de la vie sociale, ces avancées vers l'égalité étant perçues comme menaçantes pour un ordre social dont l'équilibre est fondé sur la hiérarchie sexuelle et la domination masculine. [Comme le sexisme,] l'antiféminisme tire sa légitimité d'une idéologie naturaliste latente qui continue de sanctionner l'idée que les hommes sont supérieurs aux femmes et qu'ils ont droit, en conséquence, à des pouvoirs et à des privilèges sur la base de leur sexe.

(Descarries 2005 : 142-143)

Comme suggéré dans l'introduction de cette contribution, on peut corrélativement considérer que l'on assiste, en ce début de xxI ${ }^{\mathrm{e}}$ siècle et sous l'influence de cet antiféminisme ordinaire, à une électoralisation du féminisme. Comme le souligne Guionnet, "les problématiques en lien avec le genre et le féminisme» sont largement diffusées auprès du grand public (2017: 122). Valeurs et principes féministes sont même désormais plus ou moins " cotés ", et l'opposition entre féminisme et antiféminisme est assourdie par l'opposition entre féminisme «à la mode» et «féminisme has been » qui permet à celles et ceux qui investissent le premier de «se placer ». En vient ainsi à être proposé, par exemple, un « féminisme light ». L'édition du magazine Elle du 3 mars 2017 titrait en Une: «Pop, léger, décomplexé... Le nouvel élan féministe ». L'édito de ce numéro est une illustration d'une mise en discours apolitique d'un rapport au féminisme 
assumé comme non élucidé, façonné par un parcours de vie et une détermination socioculturelle idiosyncrasiques :

J'ai longtemps été une féministe complexée. Et du coup silencieuse. Complexée parce que les femmes de mon entourage portant ce combat fièrement et avec une urgence infatigable le plaçaient dans une telle sphère intellectuelle et acharnée que je ne m'y sentais pas légitime. Ou bien tellement agressive que je le fuyais. De peur. Sans être complètement dépourvue de cerveau ni de courage, je pressentais bien que, si j'y mettais les pieds, j'allais être happée par un vortex lourd et en colère qui m'obligerait à ne pas être tout à fait moi-même. En marche forcée... Convaincue que le féminisme était obligatoirement usant. Néanmoins bien opportuniste... Je clamais ma reconnaissance éternelle à ces militantes de faire le job, pour moi, ma sueur, mes amies, ma fille. Et puis Trump est arrivé. [...] le 21 janvier, j'ai posé mon café latte, enfilé mes Nike, et j'ai rejoint la Women's March du Trocadéro organisée en parallèle de celle de Washington. Mon éveil, mon déclic... Cette marche engagée, solidaire, exaltait le plus bel exemple de solidarité féminine qui soit. C'était la première fois que je manifestais pour les droits des femmes. La première fois que je manifestais tout court, d'ailleurs. J'y suis allée sans pancarte, sans trompette. Mais j'étais là. En présence silencieuse. [...] Puis, je suis retournée à mes occupations et à mon « slacktivisme » qui me pousse, de temps en temps, mais sans forcer, non loin de mon canapé, à poster ici et là un mantra féministe, un like ou à partager sur Facebook un article faisant avancer la cause. Parfaitement satisfaite de ce féminisme occasionnel, dilettante, light, cool, appelez-le comme vous voulez, mais dont toutes les études montrent qu'il est utile. Sa résonance et son influence tiennent à la formidable vitalité des réseaux sociaux touchant une foule inestimable de femmes et d'hommes. Et je ne me pose plus ces questions : suis-je assez féministe si je ne sors pas les griffes systématiquement à la première inégalité hommesfemmes? Suis-je assez féministe si je considère comme quasi orgasmique qu'un homme me tienne la porte, tire la chaise avant que je ne m'assoie? Suis-je assez féministe si, parfois (aveu honteux), je juge les autres femmes sur leurs vêtements? La réponse est oui. Parallèlement au « Nous devrions tous être féministes » arboré par la merveilleuse Virginie Efira en couverture de ce numéro spécial, je dirais, surtout, que nous pouvons tous être féministes. À la dose que l'on souhaite. (Erin Doherty, « L'édito »)

28 Une telle prise de position est emblématique d'un rapport « décomplexé » au féminisme, de performances féministes individuelles libérées de tout formatage, idéologique et/ou pragmatique, de toute « dette » à l'égard de mouvements pionniers.

La prise en compte de discours ordinaires de ce genre, contrastant avec d'autres, situés théoriquement et politiquement, considérés comme "légitimes » ou " autorisés ", nous semble indispensable pour provoquer un «choc», faire se rencontrer des voix contradictoires, et permettre de réfléchir aux modalités de négociation et de mise en discours des rapports contemporains au féminisme. À cet égard, pour Détrez, " mieux vaut que le féminisme soit à la mode que de considérer le mot comme une insulte » (cité dans Leportois 2017). De mon point de vue, tout en étant réjouissant, ce foisonnement d'élans feminist-friendly light, dé-politisés, peut en même temps induire une perte de sens, une désincarnation et un désinvestissement potentiellement tout aussi préjudiciables au féminisme que des formes explicites d'antiféminisme. Mais comment décrire ce risque sans retomber dans une apologie d'un féminisme "comme il faut "? Dans son essai Penser dans un monde mauvais, De Lagasnerie propose un décalage qui semble judicieux :

Les questions «qu'est-ce que la philosophie?» ou «quelle méthode pour les sciences sociales?» n'ont pas de sens. Il faut rompre avec ces modes de questionnements et ces formulations pour poser les questions autrement. Il faut 
toujours nous demander : "qu'est-ce que la philosophie dans un monde mauvais?» ou, mieux encore, "Comment faire des sciences sociales dans ce monde-là, à ce moment-là? »(De Lagasnerie 2017 : 21)

Il me semble en aller de même pour ce qui est du féminisme. Plutôt que de se demander "ce que c'est », ou dans quel courant il s'incarne, on peut se concentrer sur comment on peut l'être dans ce monde «mauvais ». Réduire le féminisme à un argument revient à lui faire perdre toute force de subversion et à risquer un conformisme stérile. Comme les sexes, les genres et les assignations qui leur sont attachées, le féminisme est à problématiser et à repolitiser en situant dans des contextes socioculturels et des parcours de vie les appropriations complexes qu'il produit. Pour intégrer cette prise en compte du "rapport au féminisme », et pour que l'imagination de chacun trouve sa place dans la création de rapports au féminisme singuliers, libérés de voix collectives sclérosantes, l'expression «se dire féministe» gagnerait certainement à être remplacée par l'expression «se parler féministe ».

\section{BIBLIOGRAPHIE}

Anderson, Elizabeth, 2015, «Feminist Epistemology and Philosophy of Science », The Stanford Encyclopedia of Philosophy, [En ligne], http://plato.stanford.edu/entries/feminism-epistemology/, consulté le $1^{\mathrm{er}}$ juin 2016.

Aronson, Pamela, 2015, «Féministes ou postféministes ? Les jeunes femmes, le féminisme et les rapports de genre », Politix, n 109, p. 135-158.

Badinter, Élisabeth, 2010, Le Conflit, la femme et la mère, Paris, Flammarion.

Barré de Miniac, Christine, 2015, Le Rapport à l'écriture. Aspects théoriques et didactiques. Nouvelle édition revue et augmentée, Villeneuve d'Ascq, Presses Universitaires du Septentrion.

Cossy, Valérie, Malbois, Fabienne, Parini, Lorena et Ricci Lempen, Silvia, 2009, « Imaginaires collectifs et reconfiguration du féminisme ", Nouvelles questions féministes. Figures du féminin dans les industries culturelles contemporaines, vol. $28, \mathrm{n}^{\circ} 1, \mathrm{p} .4-12$.

De Lagasnerie Geoffroy, 2017, Penser dans un monde mauvais, Paris, PUF.

Descarries, Francine, 2005, «L'antiféminisme “ordinaire” ", Recherches féministes, vol. 18, n², p. 137-151.

Dupuis-Déri, Francis et Lamoureux, Diane (dir.), 2015, Les Antiféminismes : analyse d'un discours réactionnaire, Montréal, Les Éditions du Remue-ménage.

Fakhry, Pascale, 2012, «Femme active et femme au foyer dans le woman's horror film américain des années 2000 : ambivalences, contradictions et impasse post-féministe ", Cinémas : revue d'études cinématographiques / Cinémas: Journal of Film Studies, vol. 22, nº 2-3, p. 61-79.

Fraisse, Geneviève, 2008, « À Contre-Temps », Genre \& Histoire, nº 2, [En ligne], http:// journals.openedition.org/genrehistoire/233.

Gracq, Julien, 1964, La Littérature à l'estomac, Utrecht, Jean-Jacques Pauvert éditeur. 
Guionnet, Christine, 2017, «Troubles dans le féminisme. Le web, support d'une zone grise entre féminisme et antiféminisme ordinaire », Réseaux, n² 201, p. 115-146.

Haraway, Donna, 1988, « Situated Knowledges: The Science Question in Feminism and the Privilege of Partial Perspective », Feminist Studies, vol. 14, n 3, p. 575-599.

Le Brun, Annie, 1977, Lâchez tout, Paris, Le Sagittaire.

Le Brun, Annie, 1990, Vagit-prop, Lâchez tout et autres textes, Paris, Ramsay, J. J. Pauvert.

Leportois, Daphnée, 2017, « Va-t-on enfin réhabituer nos yeux à des corps normaux ? ", Slate, 15 avril 2017, [En ligne], http://www.slate.fr/story/143558/rehabituer-corps-normaux.

Macé, Éric, 2003, « Le piège de la “cause des femmes”. Éléments pour un mouvement antisexiste post-féministe », Cosmopolitiques, $\mathrm{n}^{\circ} 4$, p. 84-103.

Nahoum-Grappe, Véronique, 1987, « Homme, femme, femme, femme, dans le petit Robert », Autrement, $\mathrm{n}^{\circ}$ 91, p. 23-25.

Preciado, Paul B., 2014, « Féminisme amnésique », Libération, 9 mai 2014, [En ligne], http:// www.liberation.fr/france/2014/05/09/feminisme-amnesique_1014052, consulté le 5 mai 2017.

Riot-Sarcey, Michèle, 2002, Histoire du féminisme, Paris, La Découverte.

Rochefort, Florence, 1998, «L'antiféminisme : un nouveau champ de recherche ", Vingtième siècle. Revue d'histoire, $\mathrm{n}^{\circ}$ 57, p. 146-147.

Skeggs, Beverley, 2015, « Des féminités ambivalentes », Des femmes respectables. Classe et genre en milieu populaire, Marseille, Agone, p. 193-232.

Zemmour, Éric, 2006, Le Premier Sexe, Paris, Denoël.

Wittig, Monique, 2013, La Pensée straight, Paris, Éditions Amsterdam.

\section{NOTES}

1. http://time.com/3576870/worst-words-poll-2014/.

2. http://time.com/3576870/worst-words-poll-2014/.

3. Pour un historique détaillé du terme, voir par exemple Michèle Riot-Sarcey, Histoire du féminisme (2002).

\section{RÉSUMÉS}

Cette contribution fait dialoguer théorie du discours, linguistique textuelle et études de genre. Par le biais d'une analyse discursive de quelques positionnements féministes et antiféministes, elle interroge les rapports au féminisme, envisagé comme un état à incorporer et à négocier. Elle montre que la circulation médiatique de rapports au féminisme non situés catalyse un antiféminisme ordinaire discursivement construit comme aussi naturel que la binarité des sexes. Elle émet également l'hypothèse que l'on assiste, en ce début de xxI siècle et sous l'influence de cet antiféminisme ordinaire, à une «électoralisation» du féminisme: valeurs et principes 
féministes sont plus ou moins cotés, et l'opposition entre féminisme et antiféminisme est assourdie par l'opposition entre féminisme « à la mode » et féminisme « has been ».

Cette contribution entend plus globalement argumenter que le féminisme est à problématiser et à repolitiser en situant dans des contextes socioculturels et des parcours de vie ses différentes incorporations de manière à stimuler des appropriations singulières créatives, libérées de préjugés sclérosants.

This contribution brings together discourse theory, textual linguistics and gender studies. Through a discursive analysis of some feminist and anti-feminist stances, she interrogates the representations and attitudes about feminism, considered as a state to be incorporated and negotiated. It shows that the media circulation of non-situated reports to feminism catalyzes a discursively constructed ordinary anti-feminism as natural as gender binarity. It also suggests that, at the beginning of the twenty-first century and under the influence of this ordinary antifeminism, feminism is being "electoralized": feminist values and principles are more or less rated, and the opposition between feminism and anti-feminism is deafened by the opposition between feminism "à la mode" and feminism "has been."

More generally, this contribution intends to argue that feminism should be problematized and repoliticized by situating its various forms of incorporation in socio-cultural contexts and life courses so as to stimulate creative singular appropriations, freed from sclerosing prejudices.

\section{INDEX}

Mots-clés : analyse du discours, antiféminisme, féminisme, identité, représentations

Keywords : discourse analysis, feminism, antifeminism, identity, representations

\section{AUTEUR}

\section{STÉPHANIE PAHUD}

Université de Lausanne, École de français langue étrangère (EFLE) 